\title{
Energy-Efficient Spectrum Leasing in Cognitive Relay Networks
}

\author{
Qingqing Jin, Haixia Zhang, Dongfeng Yuan, Jiali Xu \\ School of Information Science and Engineering \\ Shandong University, Jinan, Shandong, China \\ \{qingqingjin, haixia.zhang,dfyuan,xujiali\}@sdu.edu.cn
}

\author{
Cheng-Xiang Wang \\ School of Engineering and Physical Sciences \\ Heriot-Watt University, EH14 4AS, Edinburgh, UK \\ cheng-xiang.wang@hw.ac.uk
}

\begin{abstract}
In this paper, we propose an energy-efficient resource allocation algorithm to minimize the total average transmission power of secondary users (SUs) in cognitive relay networks. Each $\mathrm{SU}$ in cognitive relay networks can choose direct transmission or cooperative transmission with the help of relay nodes (RNs). The primary users (PUs) may lease the licensed spectrum for a fraction of time to SUs for some remuneration. So the SUs adopt time-division multiple-access (TDMA) to access the licensed spectrum. For energy efficiency, we jointly consider the time slot scheduling and transmission strategy selection (i.e. direct transmission or cooperative transmission) problem. Numerical results indicate that the transmission power consumption indeed decreases due to the cooperative diversity.
\end{abstract} tion;

Keywords-energy-efficiency; spectrum leasing; relay selec-

\section{INTRODUCTION}

As the spectrum scarcity becomes severe in recent years, cognitive radio (CR) technology [1] [2] has attracted more and more attention in the world. According to the awareness of PUs on the existence of SUs, existing works on CR model can be divided into two categories, Commons model and Property-right model [3]. In the first model, PUs are unaware of the presence of SUs. So SUs can access the licensed spectrum without harmful interference to PUs [4]. In the second model, PUs are aware of the presence SUs and decide to lease part of the licensed spectrum to SUs for appropriate remuneration, which is also called spectrum leasing [3] [4].

Besides the spectrum scarcity problem, the energy cost is another significant problem for wireless communications recently. So "Green Communications" is now a hot topic aiming to reduce overall energy consumption while optimizing system capacity and maintaining user Quality of Service (QoS) [5]. Especially in wireless sensor networks (WSNs), energy efficiency is a critical issue due to the fact that the sensors are normally powered by batteries which can not be replaced or recharged easily. Cooperative relay communication, due to its spatial diversity, has the advantages of reducing signal transmission power and improving data rate.

This work was supported by NSFC (No. 60832008, No. 60972403) and RCUK for the "UK-China Science Bridges: R\&D on (B)4G Wireless Mobile Communications".
So in WSNs, the sensors can save power by establishing high quality links with the help of the relay nodes (RNs).

For energy efficiency, [6] investigated the energy-efficient resource allocation over fading channels. But it didn't take advantage of the spatial diversity with RNs. For cooperative relay communication, [7] presented a joint routing, relay selection and power allocation algorithm to minimize network power consumption. But it ignored the multi-user multiple-access problem. In [8], a distributed power control algorithm was proposed for wireless relay networks to minimize the total transmission power. But it only analysed the interference channel environment.

In order to improve the spectrum efficiency and prolong the operating time of the system, we study the cognitive relay networks where the SUs are sensor pairs and multiple RNs can be selected to relay the transmission. The transmission spectrum for SUs is licensed to PUs. And PUs could lease the licensed spectrum for a fraction of time to SUs for some remuneration. Each SU chooses the transmission strategy (i.e. direct transmission or cooperative transmission) to establish high quality link. So we propose a joint time slot scheduling, relay selection and power control algorithm to minimize the total average transmission power of SUs, guaranteeing the minimum rate requirement of PUs and SUs.

The remainder of this paper is organized as follows. In Section II, we describe the system transmission model. The time slot scheduling and transmission strategy selection problem will be studied in Section III. The numerical results are presented for performance evaluation in Section IV. We conclude the whole paper in Section V.

\section{System Transmission Model}

In a cognitive relay network, we assume that there are $K$ pairs of SUs. Each pair has one transmitter and receiver. $M$ RNs are located randomly in the scope of the network. Each SU can establish its link by direct transmission or cooperative transmission with the help of a $\mathrm{RN}$ in the decode-andforward (DF) protocol. All the SUs transmit data in the same spectrum channel which is licensed to the primary user (PU). The transmission channel is assumed to be block fading channel and the instantaneous channel power gains for the direct transmission, source-relay transmission and relaydestination transmission are $\boldsymbol{g}=\left\{g_{k}\right\}=\left[g_{1}, g_{2}, \cdots, g_{K}\right]$, 
$\boldsymbol{g}_{\boldsymbol{s r}}=\left\{g_{k m}\right\}=\left[g_{11}, \cdots, g_{1 M} ; \cdots ; g_{K 1}, \cdots, g_{K M}\right]$ and $\boldsymbol{g}_{\boldsymbol{r d}}=\left\{g_{m k}\right\}=\left[g_{11}, \cdots, g_{M 1} ; \cdots ; g_{1 K}, \cdots, g_{M K}\right]$, respectively. Without loss of generality, the transmission bandwidth $B$ and the variance of AWGN $N_{o}$ at the receivers and RNs are assumed to be unit.

For the primary user network (PUN), the PUs will lease some time slots to the SUs if their average transmission rates per block are guaranteed above the minimum requirement. So we schedule the SUs to access the transmission channel in TDMA mode. In this paper, we consider one PU for simplicity and the work in this paper can be easily extended to the scenario with multiple PUs. Each user $k$ (including PU, i.e. $k=0$ ) will be scheduled to transmit over nonoverlapping fractions of time in each block, denoted as $\tau_{k}(\boldsymbol{h}) \geq 0, k=0,1, \cdots, K$. The durations satisfy $\sum_{k=0}^{K} \tau_{k}(\boldsymbol{h}) \in[0,1], \forall \boldsymbol{h}$, where $\boldsymbol{h}$ denotes the set of all the transmission channel power gains in each block.

\section{A. PU Transmission Model}

For the PU transmission, the transmit power $P_{p}$ is fixed. So the time slot allocated to the PU must be long enough to guarantee the minimum average rate requirement per block, which can be written as

$$
\tau_{0}(\boldsymbol{h}) \log _{2}\left(1+g_{0} P_{p}\right) \geq R_{\min }^{p},
$$

where $g_{0}$ is the channel power gain for PU transmission and $r_{\text {min }}^{p}$ is the minimum average rate requirement for PU. It can be rewritten equivalently as

$$
\tau_{0}(\boldsymbol{h}) \geq \frac{R_{\min }^{p}}{\log _{2}\left(1+g_{0} P_{p}\right)} .
$$

\section{B. SUs Transmission Model}

In this paper, one SU can only leverage at most one RN for cooperative transmission, because one $\mathrm{RN}$ is enough to achieve full diversity of cooperation [9].

For the direct transmission, the achieved rate $(\mathrm{b} / \mathrm{s} / \mathrm{Hz})$ of SU $k$ in each block can be given as

$$
r_{k}^{d}\left(\tau_{k}(\boldsymbol{h}), p_{k}^{s}(\boldsymbol{h})\right)=\tau_{k}(\boldsymbol{h}) \log _{2}\left(1+\frac{g_{k} p_{k}^{s}(\boldsymbol{h})}{\tau_{k}(\boldsymbol{h})}\right),
$$

where $p_{k}^{s}(\boldsymbol{h})$ is the average transmit power of SU $k$ per block.

In the DF cooperative transmission, the duration for SU transmission is divided into two equal time slots. In the first time slot, the data packets transmitted by SU transmitter will be received by $\mathrm{SU}$ receiver and $\mathrm{RN}$. The $\mathrm{RN}$ decodes the packets and forwards them to the SU receiver in the second time slot. The packets received from the SU transmitter and from the RN will be combined in the SU receiver using maximum ratio combining (MRC). The achieved rate of SU $k$ at $\mathrm{RN} m$ in the first time slot is given by

$$
r_{k}^{r}\left(\tau_{k}(\boldsymbol{h}), p_{k}^{s}(\boldsymbol{h})\right)=\frac{\tau_{k}(\boldsymbol{h})}{2} \log _{2}\left(1+\frac{g_{k m} p_{k}^{s}(\boldsymbol{h})}{\tau_{k}(\boldsymbol{h}) / 2}\right),
$$

and the achieved rate of $\mathrm{SU} k$ at the $\mathrm{SU}$ receiver in the second time slot is given by

$$
\begin{aligned}
& r_{k}^{c}\left(\tau_{k}(\boldsymbol{h}), p_{k}^{s}(\boldsymbol{h}), p_{m k}^{r}(\boldsymbol{h})\right)= \\
& \quad \frac{\tau_{k}(\boldsymbol{h})}{2} \log _{2}\left(1+\frac{g_{k} p_{k}^{s}(\boldsymbol{h})}{\tau_{k}(\boldsymbol{h}) / 2}+\frac{g_{m k} p_{m k}^{r}(\boldsymbol{h})}{\tau_{k}(\boldsymbol{h}) / 2}\right),
\end{aligned}
$$

where $p_{m k}^{r}(\boldsymbol{h})$ is the average transmit power of RN $m$ to forward the data packets for SU $k$ per block.

Note that the achieved rate at RN must be higher than that by direct transmission in the first time slot to make the cooperative transmission to be useful. Based on this condition, it is easy to find that $g_{k m}$ must be higher than $g_{k}$, i.e. $g_{k m} \geq g_{k}$. This condition will also limit the search process for the best RN selection. For energy-efficient communications, the transmit powers of SU and RN should be controlled to make the achieved rates of source-relay transmission and cooperative transmission be equal, i.e.,

$$
r_{k}^{r}\left(\tau_{k}(\boldsymbol{h}), p_{k}^{s}(\boldsymbol{h})\right)=r_{k}^{c}\left(\tau_{k}(\boldsymbol{h}), p_{k}^{s}(\boldsymbol{h}), p_{m k}^{r}(\boldsymbol{h})\right) .
$$

Based on (4) and (5), this condition is equivalent to

$$
g_{k m} p_{k}^{s}(\boldsymbol{h})=g_{k} p_{k}^{s}(\boldsymbol{h})+g_{m k} p_{m k}^{r}(\boldsymbol{h}) .
$$

From the analysis above, the achieved rate of SU per block depends on not only the transmit powers and channel power gains but also the different transmission strategies (i.e. direct transmission or cooperative transmission). Let $r_{k}\left(\tau_{k}(\boldsymbol{h}), p_{k}(\boldsymbol{h})\right)$ denote the achieved rate of SU $k$ per block for different transmission strategies. Due to the energy efficiency condition (6) for the cooperative transmission, the achieved rate can be given as

$$
\begin{aligned}
& r_{k}\left(\tau_{k}(\boldsymbol{h}), p_{k}(\boldsymbol{h})\right)= \\
& \left\{\begin{array}{cl}
r_{k}^{d}\left(\tau_{k}(\boldsymbol{h}), p_{k}^{s}(\boldsymbol{h})\right), & \text { Direct transmission } \\
r_{k}^{r}\left(\tau_{k}(\boldsymbol{h}), p_{k}^{s}(\boldsymbol{h})\right), & \text { Cooperative transmission. }
\end{array}\right.
\end{aligned}
$$

where $p_{k}(\boldsymbol{h})$ denotes the average transmission power of SU. For different transmission strategies, $p_{k}(\boldsymbol{h})$ can be written as

$p_{k}(\boldsymbol{h})= \begin{cases}p_{k}^{s}(\boldsymbol{h}), & \text { Direct transmission } \\ p_{k}^{s}(\boldsymbol{h})+p_{m k}^{r}(\boldsymbol{h}), & \text { Cooperative transmission }\end{cases}$

Due to the expression (8), we can see that $r_{k}\left(\tau_{k}(\boldsymbol{h}), p_{k}(\boldsymbol{h})\right)$ has no relationship with $p_{m k}^{r}(\boldsymbol{h})$. So $r_{k}\left(\tau_{k}(\boldsymbol{h}), p_{k}(\boldsymbol{h})\right)$ can be rewritten as $r_{k}\left(\tau_{k}(\boldsymbol{h}), p_{k}^{s}(\boldsymbol{h})\right)$.

\section{PROBlem Formulation AND OPtimization}

In this section, we study the joint time slot scheduling, transmission strategy selection and power control problem using the convex optimization method. Based on the rate requirements of the $\mathrm{PU}$ and SUs, the problem can be formulated as following 


$$
\begin{array}{ll}
\min & \sum_{k=1}^{K} \mathbb{E}_{\boldsymbol{h}}\left[p_{k}(\boldsymbol{h})\right] \\
\text { s.t. } & \mathbb{E}_{\boldsymbol{h}}\left[r_{k}\left(\tau_{k}(\boldsymbol{h}), p_{k}^{s}(\boldsymbol{h})\right)\right] \geq R_{\text {min }}^{s}, \quad k \in[1, K](11) \\
& \tau_{0}(\boldsymbol{h})+\sum_{k=1}^{K} \tau_{k}(\boldsymbol{h}) \leq 1,
\end{array}
$$

where $R_{\text {min }}^{s}$ is the minimum rate requirement for each SU transmission. $\mathbb{E}_{\boldsymbol{h}}[\cdot]$ denotes the expectation of channel power gain vector $h$.

First, we introduce the lagrange multipliers and relax the constraint (11). The lagrangian function is given by

$$
\begin{aligned}
L(\boldsymbol{\tau}, \boldsymbol{p}, \boldsymbol{\lambda}) & =\sum_{k=1}^{K} \mathbb{E}_{\boldsymbol{h}}\left[p_{k}(\boldsymbol{h})\right] \\
& +\sum_{k=1}^{K} \lambda_{k}\left(R_{\min }^{s}-\mathbb{E}_{\boldsymbol{h}}\left[r_{k}\left(\tau_{k}(\boldsymbol{h}), p_{k}^{s}(\boldsymbol{h})\right)\right]\right),(13) \\
& =\mathbb{E}_{\boldsymbol{h}}\left[\sum_{k=1}^{K}\left(p_{k}(\boldsymbol{h})-\lambda_{k} r_{k}\left(\tau_{k}(\boldsymbol{h}), p_{k}^{s}(\boldsymbol{h})\right)\right)\right] \\
& +R_{\min }^{s} \sum_{k=1}^{K} \lambda_{k},
\end{aligned}
$$

where $\lambda=\left[\lambda_{1}, \lambda_{2}, \cdots, \lambda_{K}\right]$ denotes the lagrange multiplier vector.

Then, the dual function which includes the time allocation constraint can be written as

$$
\begin{aligned}
D(\boldsymbol{\lambda})= & \min \quad L(\boldsymbol{\tau}, \boldsymbol{p}, \boldsymbol{\lambda}), \\
\text { s.t. } & \sum_{k=1}^{K} \tau_{k}(\boldsymbol{h}) \leq 1-\tau_{0}(\boldsymbol{h}),
\end{aligned}
$$

and the dual problem is optimized with the dual variable $\lambda$ nonnegativity constraint.

$$
\begin{array}{cl}
\max & D(\boldsymbol{\lambda}), \\
\text { s.t. } & \boldsymbol{\lambda} \geq 0,
\end{array}
$$

Due to the fact that the achieved rate of the SU in each block $r_{k}\left(\tau_{k}(\boldsymbol{h}), p_{k}^{s}(\boldsymbol{h})\right)$ is a concave function subject to $\tau_{k}(\boldsymbol{h})$ and $p_{k}^{s}(\boldsymbol{h})$, the original problem is a convex optimization problem. So the duality gap is zero [10]. That is to say, we solve the original problem equivalently by solving the dual problem.

To solve the dual problem, we should first find the dual function $D(\boldsymbol{\lambda})$ in (15). Let us define a link-cost (power minus weighted rate) function per user as

$$
\phi_{k}\left(\tau_{k}(\boldsymbol{h}), p_{k}(\boldsymbol{h}), \boldsymbol{\lambda}\right)=p_{k}(\boldsymbol{h})-\lambda_{k} r_{k}\left(\tau_{k}(\boldsymbol{h}), p_{k}^{s}(\boldsymbol{h})\right) .
$$

If $\tau(\boldsymbol{h}) \neq 0$, defining $\rho_{k}(\boldsymbol{h})=p_{k}(\boldsymbol{h}) / \tau_{k}(\boldsymbol{h})$ as the transmit power of SU $k$, the link-cost function can be rewritten as

$$
\phi_{k}\left(\tau_{k}(\boldsymbol{h}), p_{k}(\boldsymbol{h}), \boldsymbol{\lambda}\right)=\tau_{k}(\boldsymbol{h}) \phi_{k}\left(\rho_{k}(\boldsymbol{h}), \boldsymbol{\lambda}\right) .
$$

Then, the optimal time and power solutions for (15) can be obtained following the next lemma which can be proved in [6].

Lemma: For each block realization $h$, the optimal transmission power and transmit power of $S U k$ is denoted as $\rho_{k}^{*}(\boldsymbol{h})$ and $\rho_{k}^{s *}(\boldsymbol{h})$. The link quality indicator for each $S U$ is

$$
\phi_{k}^{*}(\boldsymbol{h}, \boldsymbol{\lambda})=\rho_{k}^{*}(\boldsymbol{h})-\lambda_{k} r_{k}\left(\rho_{k}^{s *}(\boldsymbol{h})\right),
$$

where $\rho_{k}^{s *}(\boldsymbol{h})=p_{k}^{s *}(\boldsymbol{h}) / \tau_{k}^{*}(\boldsymbol{h})$. Then the optimal time and power solution for (15) in each block follows a greedy policy

$\left\{\begin{array}{l}\tau_{k^{*}}^{*}(\boldsymbol{h}, \boldsymbol{\lambda})=1-\tau_{0}(\boldsymbol{h}), \quad p_{k^{*}}^{*}(\boldsymbol{h}, \boldsymbol{\lambda})=\rho_{k^{*}}^{*}(\boldsymbol{h}) \tau_{k^{*}}^{*}(\boldsymbol{h}, \boldsymbol{\lambda}), \\ \tau_{k}^{*}(\boldsymbol{h}, \boldsymbol{\lambda})=p_{k}^{*}(\boldsymbol{h}, \boldsymbol{\lambda})=0, \quad \forall k \neq k^{*}(\boldsymbol{h}, \boldsymbol{\lambda}) .\end{array}\right.$

where $k^{*}(\boldsymbol{h}, \boldsymbol{\lambda})=\arg \min \phi_{k}^{*}(\boldsymbol{h}, \boldsymbol{\lambda})$.

Note that the average transmission power of SU $p_{k}(\boldsymbol{h}, \boldsymbol{\lambda})$ has different expressions in different transmission strategies in (9). For its optimal solution $p_{k}^{*}(\boldsymbol{h}, \boldsymbol{\lambda})$, we need to solve the transmission strategy selection problem for each SU.

First, we consider the link quality indicator for each SU in different strategies. In direct transmission strategy, due to (8) and (9), we have

$$
\phi_{k}^{*}(\boldsymbol{h}, \boldsymbol{\lambda})=\rho_{k}^{s *}(\boldsymbol{h})-\lambda_{k} r_{k}^{d}\left(\rho_{k}^{s *}(\boldsymbol{h})\right) .
$$

In cooperative transmission strategy with $\mathrm{RN} m$, we have

$$
\phi_{k}^{*}(\boldsymbol{h}, \boldsymbol{\lambda})=\rho_{k}^{s *}(\boldsymbol{h})+\rho_{m k}^{r *}(\boldsymbol{h})-\lambda_{k} r_{k}^{r}\left(\rho_{k}^{s *}(\boldsymbol{h})\right),
$$

where $\rho_{m k}^{r *}(\boldsymbol{h})=p_{m k}^{r *}(\boldsymbol{h}) / \tau_{k}^{*}(\boldsymbol{h})$.

So the best transmission strategy is, given the dual variable $\lambda$, the one which leads to the smallest link quality indicator $\phi_{k}^{*}(\boldsymbol{h}, \boldsymbol{\lambda})$.

For direct transmission, $\rho_{k}(\boldsymbol{h})=\rho_{k}^{s}(\boldsymbol{h})$. Due to (19) and (20), it is easy to prove that $\phi_{k}\left(\rho_{k}^{s}(\boldsymbol{h}), \boldsymbol{\lambda}\right)$ is a convex function for $\rho_{k}^{s}(\boldsymbol{h}) \geq 0$. So $\rho_{k}^{s *}(\boldsymbol{h})$ can be obtained by equating the derivative of $\phi_{k}\left(\rho_{k}^{s}(\boldsymbol{h}), \boldsymbol{\lambda}\right)$ w.r.t. $\rho_{k}^{s}(\boldsymbol{h})$ to zero. The solution is obtained as

$$
\rho_{k}^{*}(\boldsymbol{h})=\rho_{k}^{s *}(\boldsymbol{h})=\left[\frac{\lambda_{k}}{\ln 2}-\frac{1}{g_{k}}\right]^{+},
$$

where $[X]^{+}=\max \{0, X\}$.

For cooperative transmission, $\rho_{k}(\boldsymbol{h})=\rho_{k}^{s}(\boldsymbol{h})+\rho_{m k}^{r}(\boldsymbol{h})$. $\rho_{m k}^{r}(\boldsymbol{h})$ could be substituted by $\rho_{k}^{s}(\boldsymbol{h})$ using the condition (7). So following the similar method for direct transmission, $\rho_{k}^{s *}(\boldsymbol{h})$ can be calculated as

$$
\rho_{k}^{s *}(\boldsymbol{h})=\frac{1}{2}\left[\frac{\lambda_{k} g_{m k}}{\left(g_{k m}+g_{m k}-g_{k}\right) \ln 2}-\frac{1}{g_{k m}}\right]^{+},
$$

and $\rho_{m k}^{r *}(\boldsymbol{h})$ can be calculated following

$$
\rho_{m k}^{r *}(\boldsymbol{h})=\frac{g_{k m}-g_{k}}{g_{m k}} \rho_{k}^{s *}(\boldsymbol{h}) .
$$

So $\rho_{k}^{*}(\boldsymbol{h})$ in cooperative transmission can be given as

$$
\rho_{k}^{*}(\boldsymbol{h})=\frac{1}{2}\left[\frac{\lambda_{k}}{\ln 2}-\frac{g_{k m}+g_{m k}-g_{k}}{g_{k m} g_{m k}}\right]^{+} .
$$




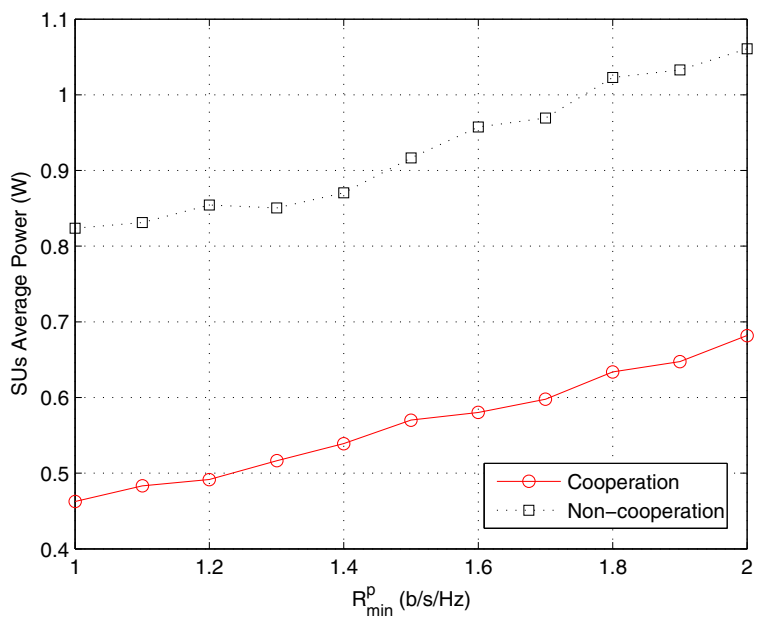

Figure 1. Comparison of cooperative and non-cooperative strategy in different $R_{\min }^{p}$.

According to the solutions in (22), the dual problem (17) can be solved using the following subgradient iteration method

$$
\begin{aligned}
\lambda_{k}(n+1) & =\left[\lambda_{k}(n)+\alpha(n)\left(R_{\min }^{s}\right.\right. \\
& \left.\left.-\mathbb{E}_{\boldsymbol{h}}\left[r_{k}\left(\tau_{k}^{*}(\boldsymbol{h}), p_{k}^{s *}(\boldsymbol{h})\right)\right]\right)\right]^{+}, \quad k \in[1, K]
\end{aligned}
$$

where $\alpha$ is the step size. It can be guaranteed that $\lambda$ converges to the optimal solutions with appropriate configuration of $\alpha$.

\section{Numerical Results}

In this section, we evaluate the proposed energy efficient resource allocation algorithm in some numerical results. We consider a cognitive relay network with 2 pairs of SUs and multiple RNs located nearby. It is assumed that there would be at least one RN in the middle of secondary transmitter and receiver. The channel power gains consider path loss with exponent 3 and Rayleigh fading distribution with unit mean. The transmit power of the PU $P_{p}$ is $10 \mathrm{~W}$ and the minimum rate required for SU $R_{\min }^{s}$ is $0.5 \mathrm{~b} / \mathrm{s} / \mathrm{Hz}$. We adopt MonteCarlo simulations to approximate the actual ergodic process of the channel power gains. All the results are obtained by averaging over 10000 blocks.

In Figure 1, we compare the required average power of SUs in cooperative and non-cooperative transmission strategies. From the figure we can see that the total average transmission power of SUs in cooperative strategy is obviously smaller than that in non-cooperative strategy. This is because that more good link channels can be chosen to save power in the cooperative strategy. Meanwhile, it is worth pointing out that the power increases as the minimum rate of the PU increases. This is due to the fact that the higher rate PU requires, the less time slots SUs can access.
So SUs should increase the power to guarantee their rate requirements.

\section{Conclusion}

In this paper, we propose an energy-efficient resource allocation algorithm to minimize the total average transmission power in cognitive relay networks. Due to the fact that the PU can lease fractions of time to SUs for transmission, SUs can access the licensed spectrum using TDMA mode. With the help of RNs, more good channels would be chosen for SUs to save power. Numerical results show that, compared with the non-cooperative transmission strategy, this algorithm indeed decreases the required transmission power which guarantees the rate requirements of PU and SUs.

\section{REFERENCES}

[1] C.-X. Wang, X. Hong, H.-H. Chen, and J. S. Thompson, "On capacity of cognitive radio networks with average interference power constraints", IEEE Trans. Wireless Commun., vol. 8, no. 4, pp. 1620-1625, Apr. 2009.

[2] C.-X. Wang, H.-H. Chen, X. Hong, and M. Guizani, "Cognitive radio network management", IEEE Vehi. Techno. Magazine, vol. 3, no. 1, pp. 28-35, Mar. 2008.

[3] O. Simeone, I. Stanojev, S. Savazzi, Y. Bar-Ness, U. Spagnolini, and R. Pickholtz, "Spectrum leasing to cooperating secondary ad hoc networks," IEEE J. Select. Areas Commun., vol. 26, no. 1, pp. 203-213, Jan. 2008.

[4] Y. Yi, J. Zhang, Q. Zhang, T. Jiang, and J. Zhang, "Cooperative Communication-Aware Spectrum Leasing in Cognitive Radio Networks," IEEE Symposium on New Frontiers in Dynamic Spectrum Access Networks (DySPAN 2010), pp. 1-11, Apr. 2010.

[5] A. Amanna, "Green Communications," Annotated Literature Review and Research Vision, Wireless@Virginia Tech.

[6] X. Wang, D. Wang, H. Zhuang, and S. D. Morgera, "Fair Energy-Efficient Resource Allocation in Wireless Sensor Networks over Fading TDMA Channels," IEEE J. Select. Areas Commun., vol. 28, no. 7, pp. 1063-1072, Sep. 2010.

[7] L. Le, E. Hossain, "Cross-Layer Optimization Frameworks for Multihop Wireless Networks Using Cooperative Diversity," IEEE Trans. Wireless Commun., vol. 7, no. 7, pp. 2592-2602, Jul. 2008.

[8] S. Zhou, H. Xiao, and Z. Niu, "Distributed Power Control for Interference-Limited Cooperative Relay Networks," IEEE International Conference on Communication (ICC 2009), pp. 1-6, Jun. 2009.

[9] Y. Zhao, R. Adve, and T. J. Lim, "Improving Amplify-andForward Relay Networks: Optimal Power Allocation versus Selection," IEEE International Symposium on Information Theory (ISIT 2006), pp. 1234-1238, 2006.

[10] S. Boyd and L. Vandenberghe, Convex Optimization. Cambridge University Press, 2004. 